\title{
PRELIMINARY VIBRATION ANALYSIS OF MAGNET/SUPPORT SYSTEM FOR 7-GEV APS STORAGE RING
}

\author{
by \\ M. W. Wambsganss \\ Materials and Components Technology Division \\ Argonne National Laboratory \\ ANL/APS/IN/VIB--89/2 \\ DE92 012379
}

As illustrated in Fig. 1 (Fig. 11.4.10-2 of Ref. 1), the storage ring quadrupoles, sextupoles, and correction magnets will be mounted on a common girder approximately $4.0 \mathrm{~m}$ long. The rigid girder, in turn, is mounted on three five-ton jacks, each of which is mounted on a pedestal that is grouted and bolted to the floor.

For this preliminary analysis, we will assume that the girder has a weight per unit length of $100 \mathrm{lb} / \mathrm{ft}$ and a bending stiffness that is significantly greater than the vertical stiffness provided by the pedestal (jack-screw) supports. This allows us to approximate the magnet/support system as a rigid beam (girder) on spring supports (jackscrews) carrying distributed masses (magnets). The model is illustrated in Fig. 2. These approximations permit us to study the rigid body translational and rotational (rocking) modes of the system. It should be noted, as illustrated in Fig. 1, that the preliminary design has two jackscrew supports at the left end and one at the right end. Therefore, if we let $k$ represent the vertical spring constant associated with a single jack-screw, $k_{1}=2 k$ (the equivalent spring constant for two springs in parallel) and $k_{2}=k$.

The model given in Fig. 2 can be further simplified to yield the model illustrated in Fig. 3, viz., a rigid body with mass $M$ and mass moment of inertia about the center of gravity $J$, mounted on two spring supports.

Using the coordinate system defined in Fig. 4, the equations of motion for the dynamic system of Fig. 3 can be written in the form

$$
\begin{aligned}
& M \ddot{x}+3 k x+k\left(a_{2}-2 a_{1}\right) \theta=0 \\
& J \ddot{\theta}+k\left(a_{2}-2 a_{1}\right) x+k\left(2 a_{1}^{2}+a_{2}^{2}\right) \theta=0
\end{aligned}
$$


Assuming harmonic motion, that is,

and

$$
x=X \cos \omega t
$$

$$
\theta=\Theta \cos \omega t
$$

allows one to determine as the system natural frequencies,

$$
\omega_{1,2}=\left\{A_{1} / 2 \pm 0.5\left(A_{1}-4 A_{2}\right)^{0.5}\right\}
$$

and, for the amplitude ratio (mode),

$$
(X / \Theta)_{1,2}=-A_{3} R\left[1-\left(\omega_{n} / \Omega\right)^{2}\right]
$$

where,

$$
\begin{aligned}
& A_{1}=1+(2 / 3) \bar{a}_{1}+(1 / 3) \bar{a}_{2} \\
& A_{2}=(2 / 9)\left(\bar{a}_{1}^{2}+\bar{a}_{2}^{2}\right) \\
& A_{3}=(1 / 3)\left(2 \bar{a}_{1}-\bar{a}_{2}\right) \\
& \Omega^{2}=3 \mathrm{k} / M \\
& R^{2}=J / M \\
& \bar{a}_{1}=a_{1} / R \\
& \bar{a}_{2}=a_{2} / R
\end{aligned}
$$

and

Further analysis requires determination of the center of mass, the total mass, and the mass moment of inertia about the center of mass for the magnet/girder system of Fig. 2. As an aid in calculating these values the $(y, z)$ coordinate system defined in Fig. 5 will be utilized. Magnet weights (as obtained from Table 11.4.1-1 of Ref.1), the esti nated girder weight, and the approximate locations of their centers of gravity (as estimated from Fig. 1) are given in Table 1.

With this information, the location of the center of mass of the magnet/girder system $\left(y_{0}, z_{0}\right)$ is calculated as 
and

$$
\mathrm{y}_{0}=6.40 \mathrm{ft} \text {, }
$$

$$
z_{0}=2.33 \mathrm{ft},
$$

and the mass $M$ and the mass moment of inertia $J$ of the magnet/girder system are calculated as

and

$$
M=426 \mathrm{lb}-\sec ^{2} / \mathrm{ft},
$$

$$
J=5850 \mathrm{lb}-\mathrm{ft}-\mathrm{sec}^{2} \text {. }
$$

Finally, we need information on the vertical stiffness of the jackscrews. In Ref. 2, this stiffness is estimated to be

$$
k \approx 10^{6} \mathrm{lb} / \mathrm{in}
$$

Substituting these values into the equations developed above give, for the natural frequencies,

and

$$
\omega_{1}=139 \mathrm{rad} / \mathrm{sec} \rightarrow f_{1}=22.1 \mathrm{~Hz},
$$

$$
\omega_{2}=361 \mathrm{rad} / \mathrm{sec} \rightarrow f_{2}=57.4 \mathrm{~Hz} \text {, }
$$

and, for the modes,

and

$$
(\mathrm{X} / \Theta)_{1}=-1.21 \mathrm{ft} / \mathrm{rad}
$$

$$
(\mathrm{X} / \Theta)_{2}=1.73 \mathrm{ft} / \mathrm{rad}
$$

The vibration modes are illustrated in Fig. 6.

It may be advantageous to design the magnet/girder/support system such that the lowest natural frequency is maximized; an additional consideration would be to design the support system such that the rlatural frequencies are not close to known excitation frequencies, thereby, avoiding a resonance condition with its associated amplification of motion. Assuming that the magnet/girder system is fixed, the design parameters that can be varied are the locations of the jack-screw supports.

The lowest coupled mode frequency is always less than the lowest of the two uncoupled mode frequencies. Consequently, the lowest mode 
frequency is greatest when the translational and rotational modes are uncoupled. From the equations of motion it can be observed that the translational and rotational modes are uncoupled when $2 a_{1}=a$. Letting $b=2 a_{1}=a_{2}$, the uncoupled equations of motion can be written as

$$
\begin{aligned}
& M \ddot{x}+3 k x=0 \\
& J \ddot{\theta}+(3 / 2) k b^{2} \theta=0
\end{aligned}
$$

By inspection, the translational mode natural frequency is

$$
f_{T}=(1 / 2 \pi) \sqrt{3 k / M}
$$

and the rotational (rocking) mode frequency can be written as

$$
f_{R}=(1 / 2)(b / R) f_{T}
$$

where, again, $J=M R^{2}$. With $M=426 \mathrm{lb}-\sec ^{2} / \mathrm{ft}$ and $R=3.70 \mathrm{ft}$, we have

and

$$
f_{T}=46.3 \mathrm{~Hz}
$$

$$
f_{R}=8.85 b ~ H z, \quad b \approx f t
$$

The maximum value of $b$ is $6.70 \mathrm{ft}$. This gives as the maximum value of the rotational mode natural frequency

$$
\left(f_{R}\right)_{\text {Max }}=59.3 \mathrm{~Hz}
$$

It should be noted that

$$
f_{R} \geq f_{T}=46.3 \mathrm{~Hz}
$$

if $b>2 R=5.23 \mathrm{ft}$. It follows that for $b=5.23 \mathrm{ft}$, the location of the jack-screws (springs) with respect to the center of mass of the system, requires $a_{1}=2.62 \mathrm{ft}$ and $a_{2}=5.23 \mathrm{ft}$.

This preliminary analysis supplements that of Ref. 2, and is intended to provide additional insights into the expected dynamic behavior of the APS magnet/support system, including coupling of translational and rocking motion, as represented by the natural frequencies and corresponding modes. The analysis also serves to 
illustrate how varying the location of the pedestal supports with respect to the system center of mass can be used to uncouple the translational and rocking modes and, thereby, to maximize the system's lowest natural frequency.

It should be noted that the magnet/support system studied is only a conceptual design as given in Ref. 1 . In actuality, there are five different magnet/support configurations comprising each of the 40 sectors of the storage ring. Also, it is expected that the details of the final design, in particular with respect to the positioning of the pedestal supports, will be somewhat different.

It should further be noted that a major assumption in the analysis is that the stiffness of the support beam with respect to the stiffness of the jack-screw supports is such that the support girder can be assumed rigid. To evaluate this assumption it is planned to perform a finite element analysis of the magnet/support system in which the flexibility of the support girder is included in the dynamic analysis.

\section{REFERENCES}

1. 7-GeV Advanced Photon Source Conceptual Design Report, ANL-87-15, April 1987

2. Chou, W. ANL Report, Light Source Note LS-77, January 1987 
Table 1 Magnet/Girder System Parameters

\begin{tabular}{llrrr}
\hline$k$ & Component & $W_{k}(\mathrm{lb})$ & $y_{k}(\mathrm{ft})$ & $z_{k}(\mathrm{ft})$ \\
\hline & & & & \\
1 & Magnet Q4 & 2,537 & 1.18 & 2.52 \\
2 & Magnet Q5 & 3,028 & 4.24 & 2.52 \\
3 & Magnet SF & 1,276 & 6.37 & 2.52 \\
4 & Magnet Q5 & 3,028 & 8.52 & 2.52 \\
5 & Magnet Q4 & 2,537 & 11.58 & 2.52 \\
$G$ & Girder & 1,350 & 6.56 & 0.50 \\
\hline
\end{tabular}


)

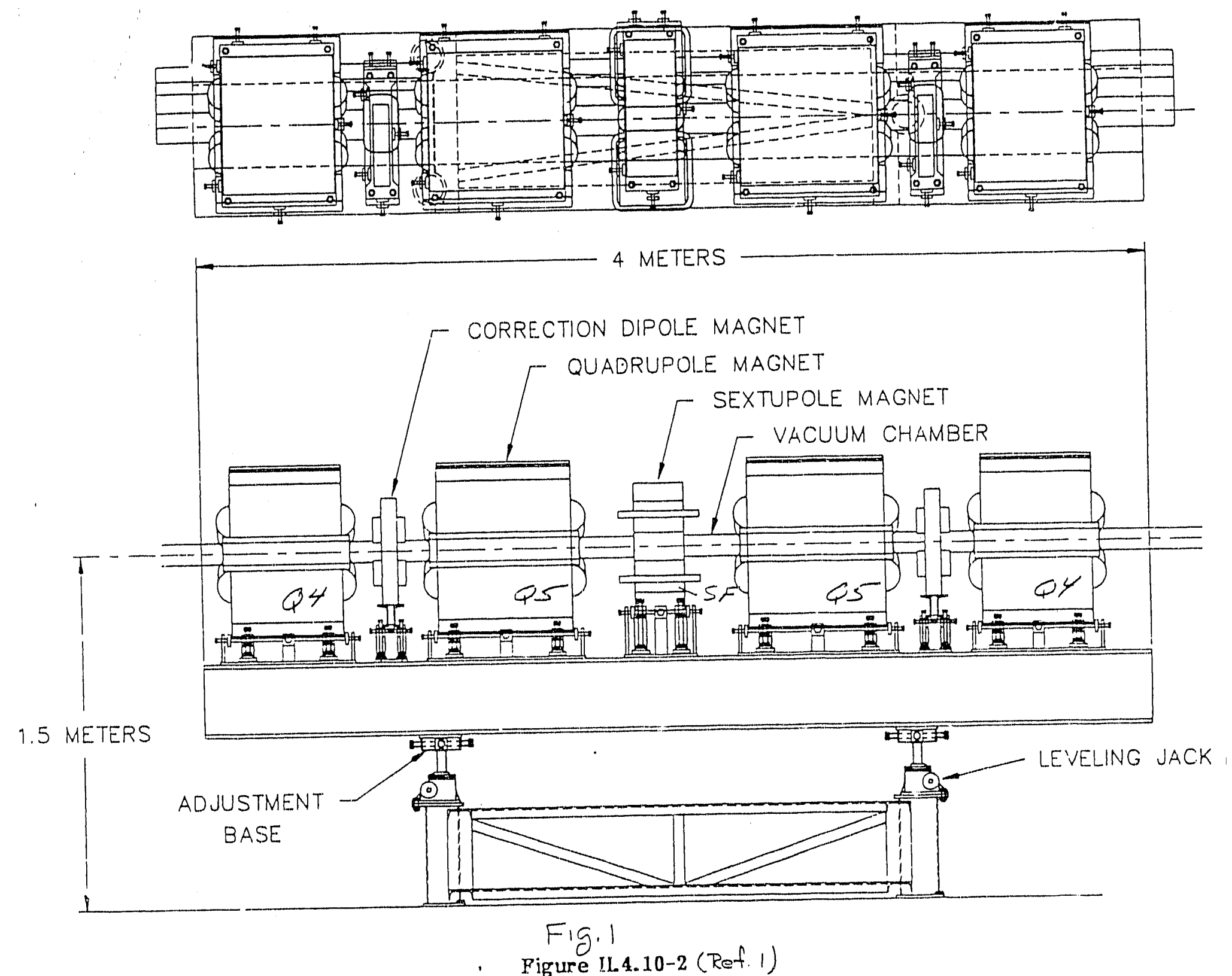

Support for the storage ring quadrupoles, sextupoles, and correction dipoles. 


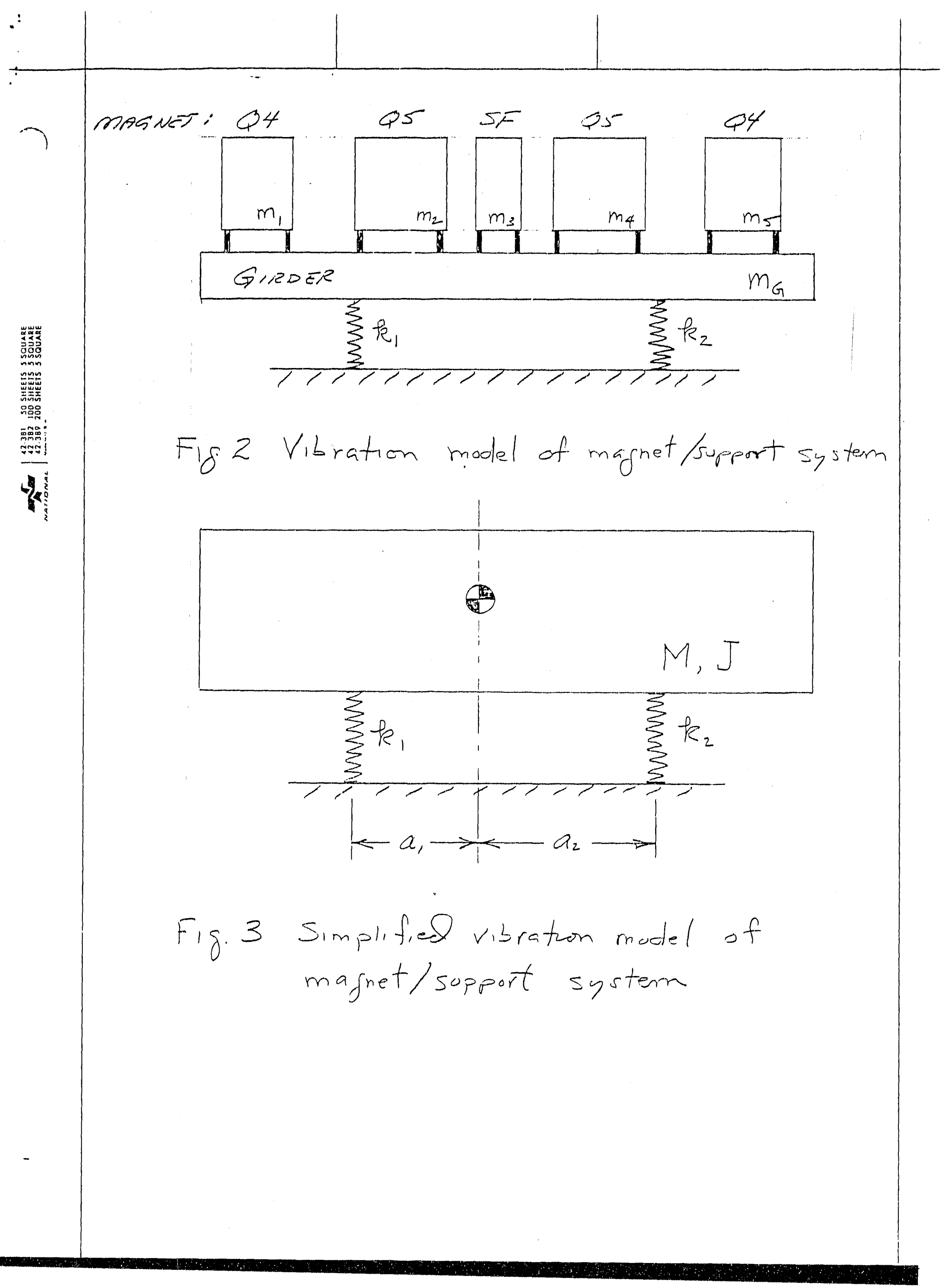



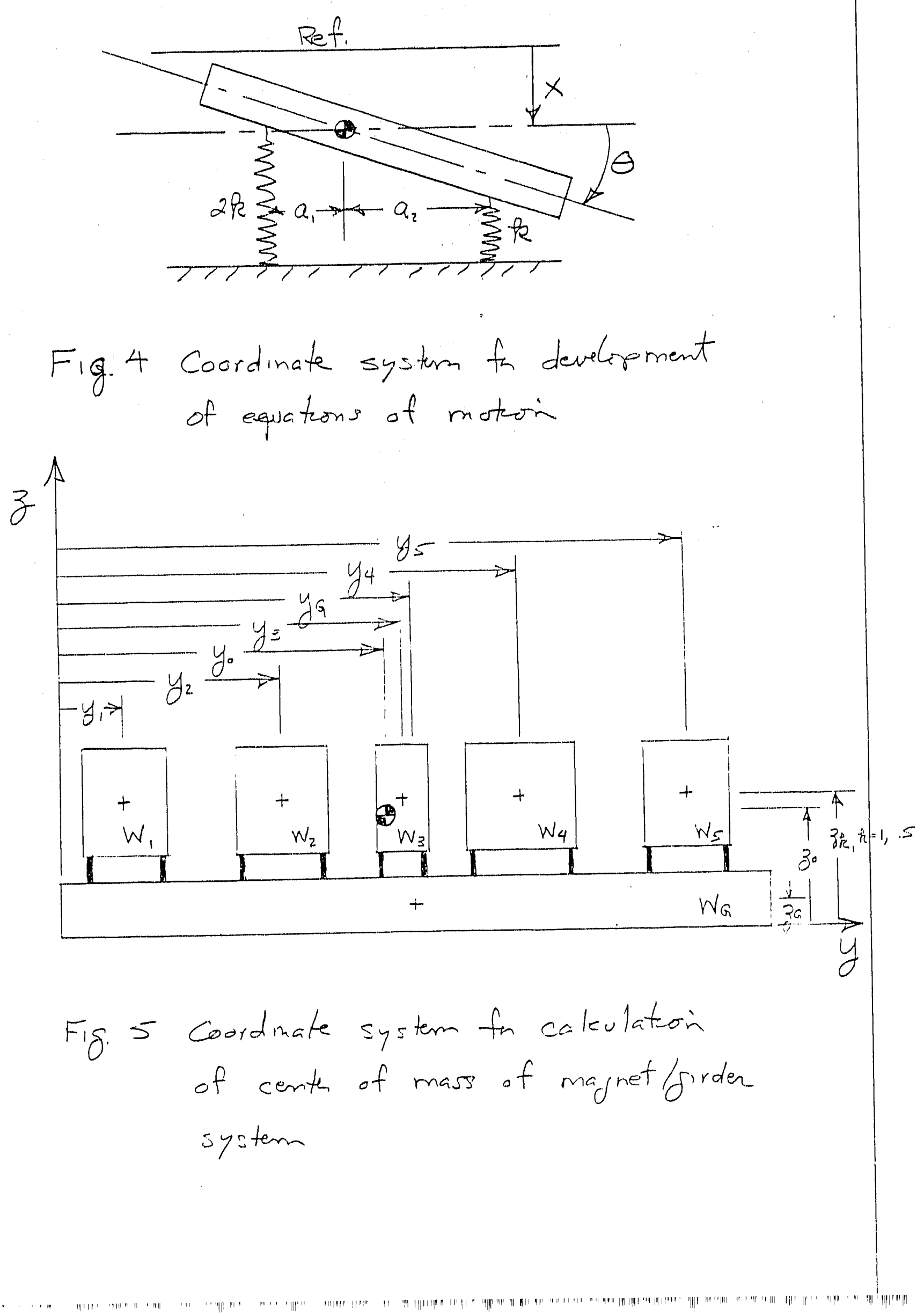


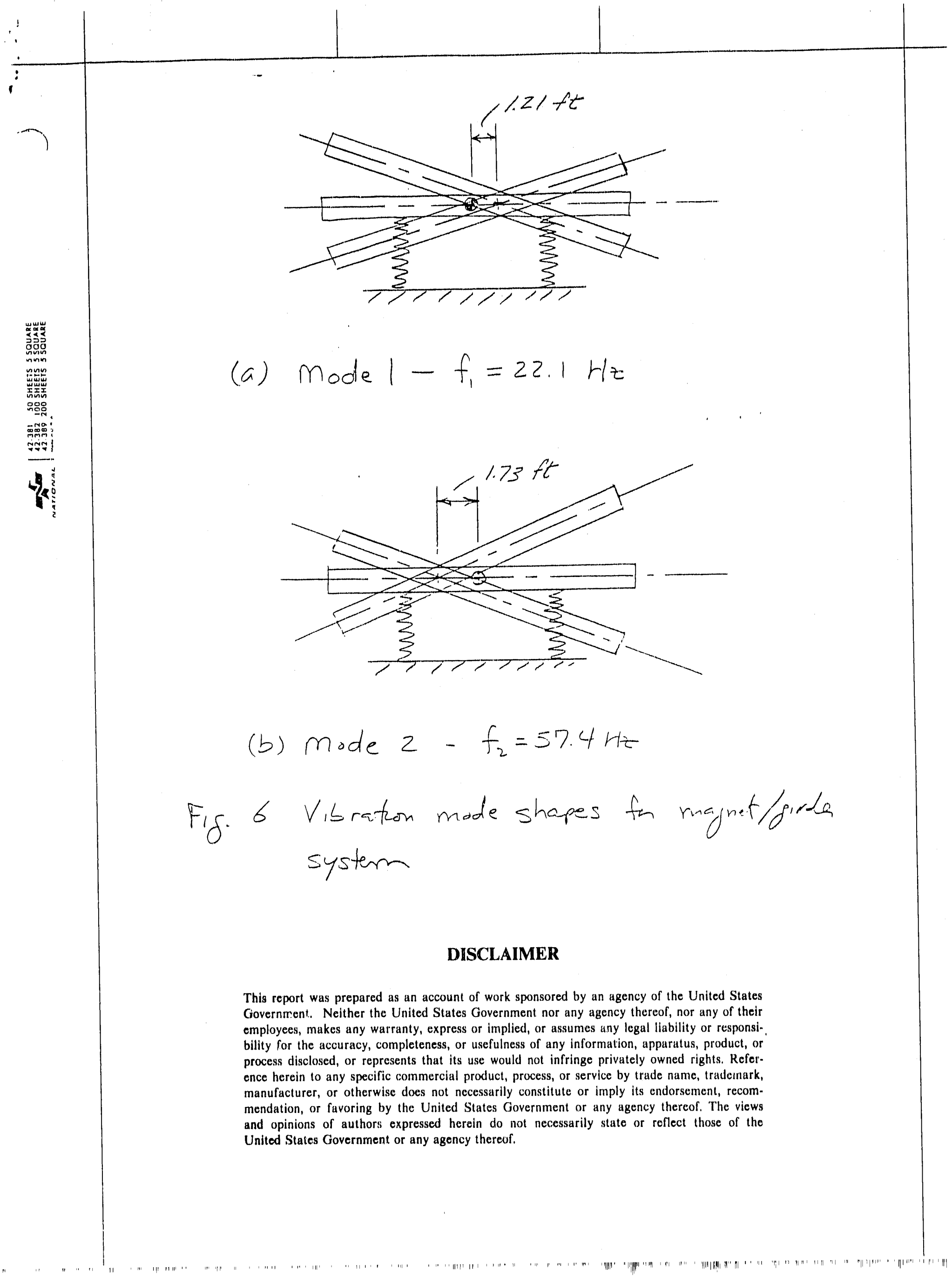



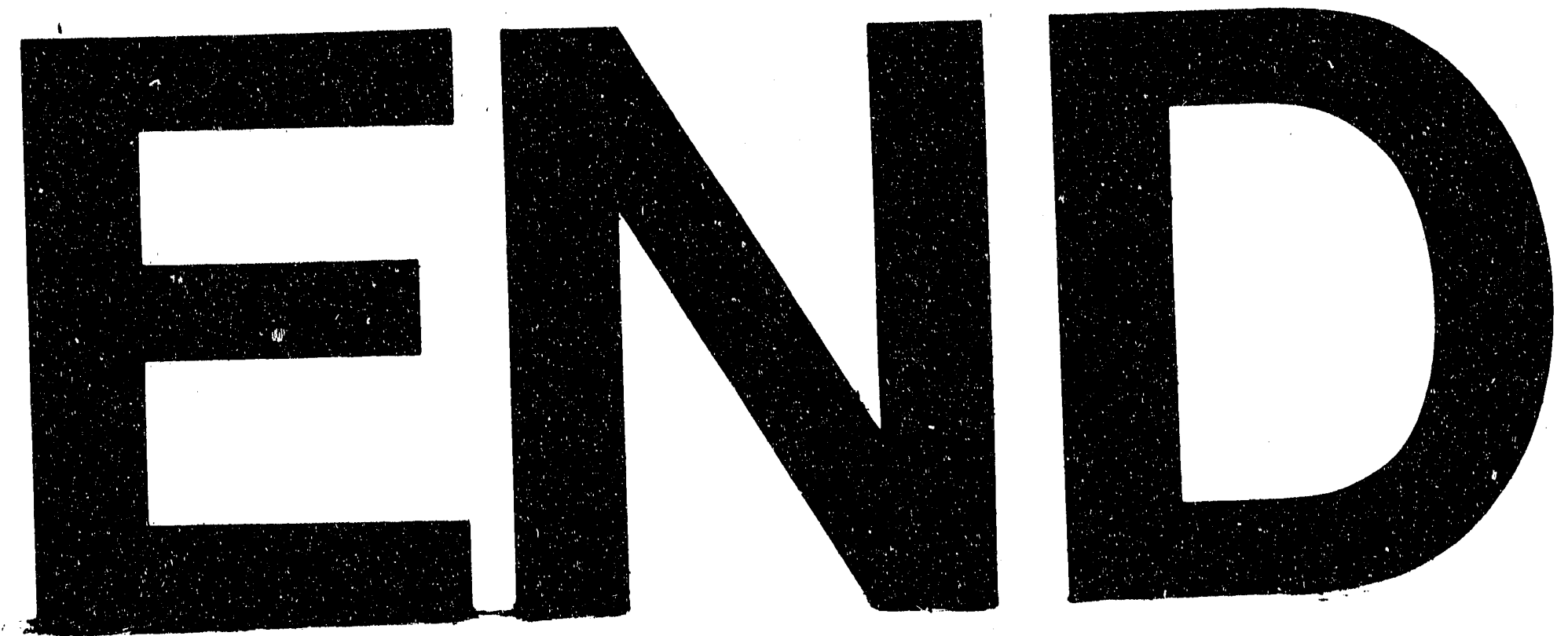

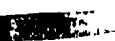
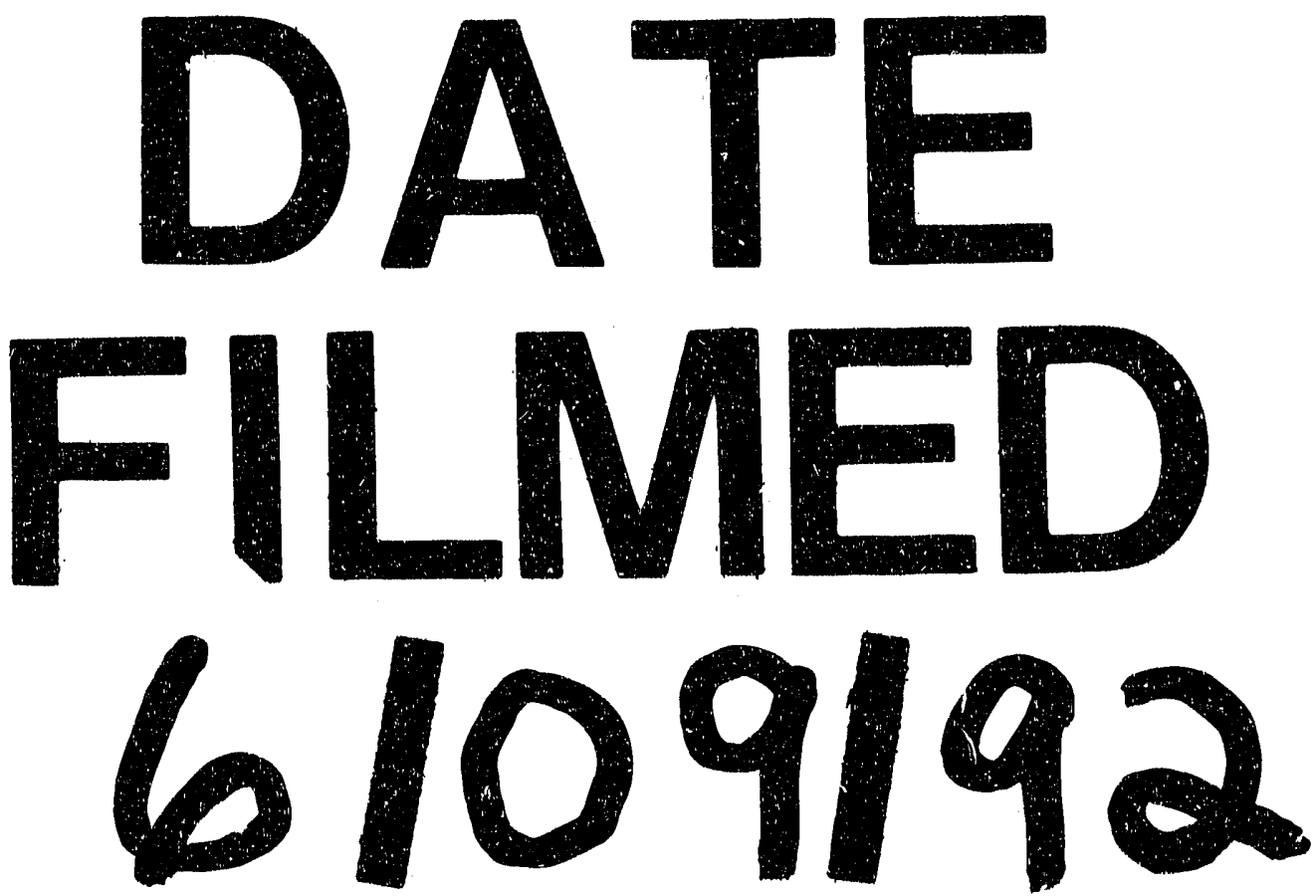


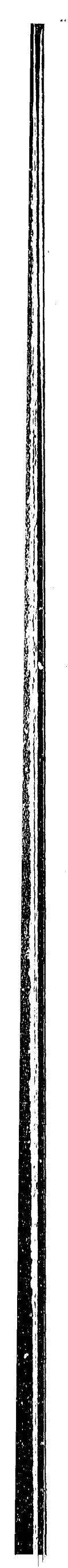

